\title{
Metachronous malignant tumors in ipsilateral salivary glands
}

\author{
Hyo Jeong Kwon, \\ Seong Ae Kim, \\ Jong Won Rhie, \\ Suk-Ho Moon \\ Department of Plastic and \\ Reconstructive Surgery, College of \\ Medicine, The Catholic University of \\ Korea, Seoul, Korea
}

\begin{abstract}
Salivary gland tumors usually appear in solitary mass in single salivary gland. The coexistence of tumors with different histological types occurring within a unilateral parotid gland is an extremely rare event. We experienced a case which two different types of malignant tumors developed at different time points in same gland; metachronous tumors. The second tumor was excised widely and reconstruction was performed by free tissue transfer. Sensory and motor nerve to the left cheek appeared to be intact, and circulation was adequate. This rare case was presented in this article.
\end{abstract}

Keywords: Adenocarcinoma / Neoplasm, second primary / Salivary gland

\section{INTRODUCTION}

Although salivary gland tumors typically appear in the form of a singular mass restricted to a unilateral salivary gland, there are extremely rare cases that involve the development and coexistence of tumors with different characteristics [1]. The coexistence of tumors with different histological types occurring within a unilateral parotid gland is an extremely rare event, and found in $<0.3 \%$ of all salivary gland neoplasms [2]. Although rarely observed, there have been cases of multiple tumor development in the salivary glands, which are mostly synchronous tumors. Synchronous tumors are defined by the development of $\geq 2$ tumors at the same time, while metachronous tumors develop at different time points.

Ipsilateral development of metachronous malignant tumors of two different types is an extremely rare event and, to the best of our knowledge, there are few, if any reports in the literature describing this entity. In the present article, we report our experi-

Correspondence: Suk-Ho Moon

Department of Plastic and Reconstructive Surgery, Seoul St. Mary's Hospital, College of Medicine, The Catholic University of Korea, 222 Banpo-daero, Seochogu, Seoul 06591, Korea

E-mail: nasuko@catholic.ac.kr

Received September 2, 2019 / Revised October 17, 2019 / Accepted November 5, 2019 ence with a patient who had been previously diagnosed with a single malignant tumor in the parotid gland and underwent parotidectomy, but was diagnosed with a different malignant tumor at the same location approximately 20 years later, which we introduce under the concept of multiple tumors.

\section{CASE REPORT}

A 69-year-old female had been visiting the authors' center for approximately 1 year with a protruding mass on the left cheek area, which had been growing in size (Fig. 1). The patient had also developed a similar left cheek mass approximately 20 years previously, which was suspected to be benign and was excised at a different center. The follow-up biopsy indicated that the mass was an epithelial-myoepithelial carcinoma (EMC)-a type of malignant tumor; consequently, the patient underwent additional total parotidectomy. The patient was determined to be in complete remission and was lost to follow-up without requiring additional treatment.

Approximately 20 years later, however, the patient visited the Seoul St. Mary's Hospital with a mass on the left cheek area that had been growing in size over the past year. From physical examination, a firm, non-tender, irregular shape mass, approxi- 

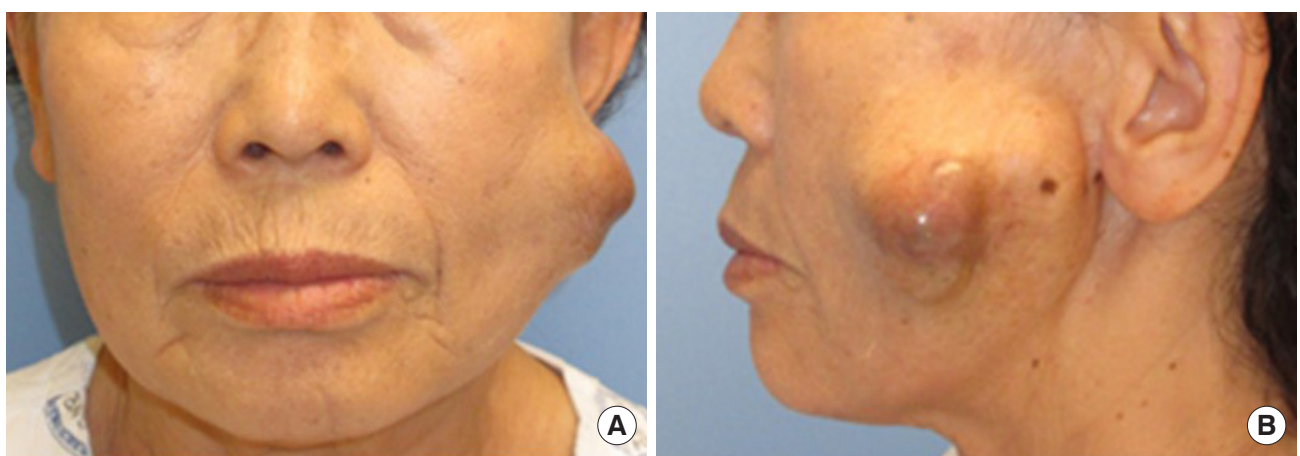

Fig. 1. Preoperative clinical photographs. (A) Anterior view and (B) lateral view.
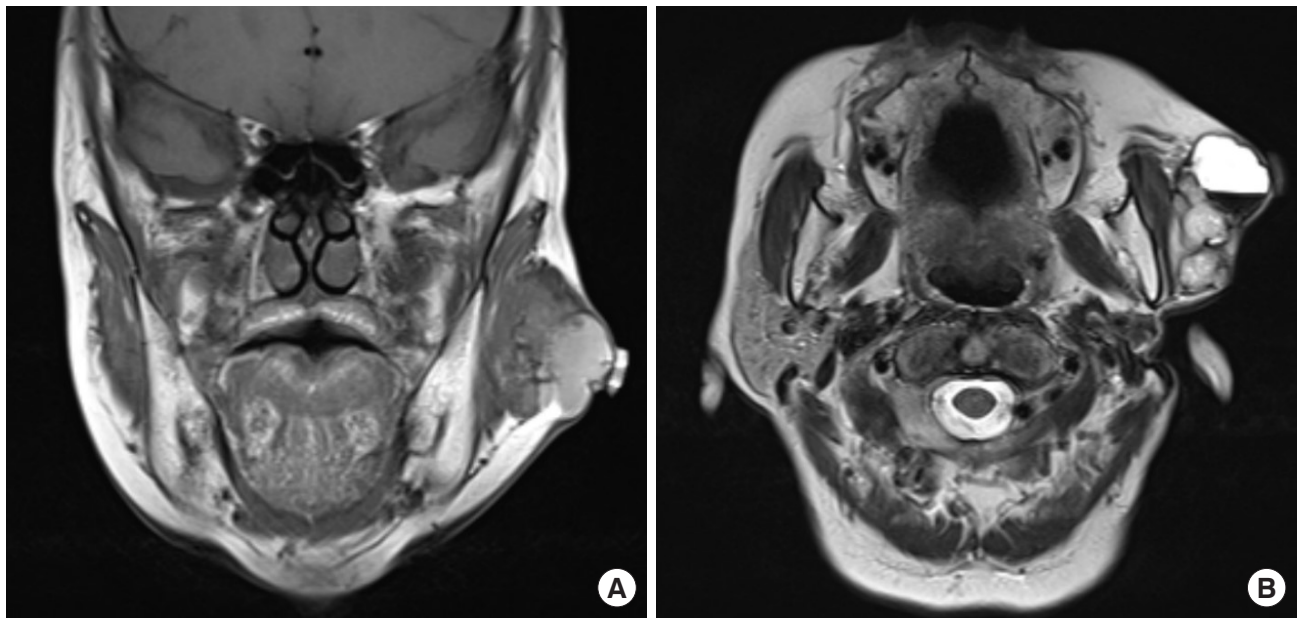

Fig. 2. Magnetic resonance imaging. (A) T1, coronal and (B) T2, axial.
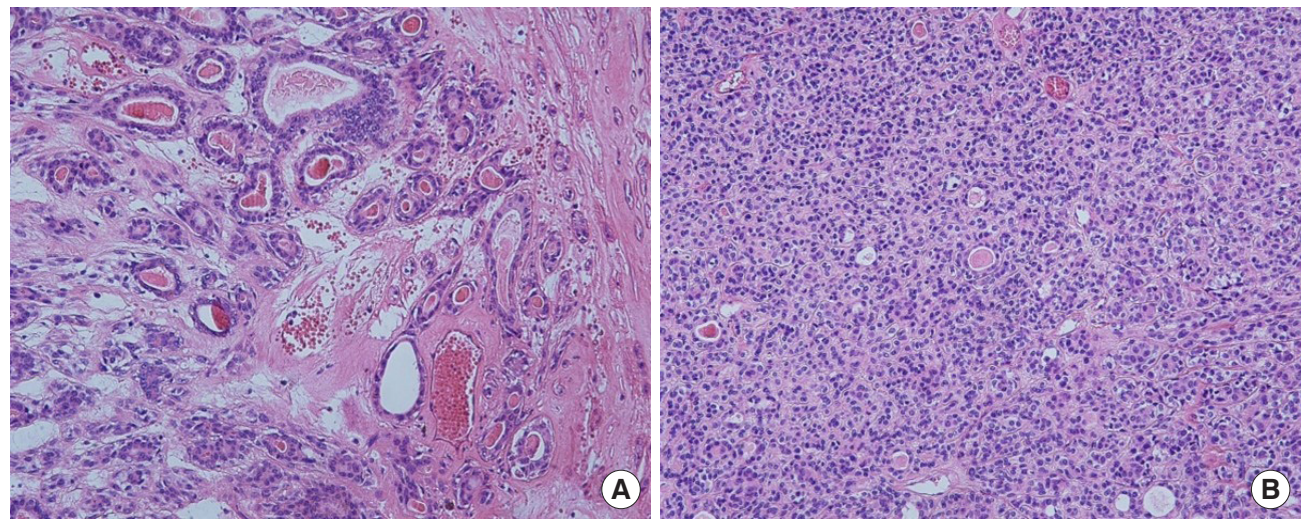

Fig. 3. Histologic findings. (A) A basal cell adenocarcinoma that exhibits invasive growth with associated perineural invasion (H\&E, $\times 100)$. (B) The tumor is composed of basaloid cells, which occur concomitantly with vague two-cell morphologies (H\&E, $\times 40)$.

mately $6 \times 6 \mathrm{~cm}$ in size, was palpated; her facial nerve function was intact. Furthermore, there was an accompanying discolored skin lesion in the upper part of the mass. Preoperative magnetic resonance imaging confirmed the presence of a welldefined mass, $2.6 \times 4.6 \times 4.6 \mathrm{~cm}$ in size, in the left masticator space, which consisted of solid and cystic parts with infiltration from the masticator muscle to the dermal layer (Fig. 2). Gross total resection of the lesion was performed with a safety margin of $1 \mathrm{~cm}$, followed by reconstruction of the wound with exposed facial nerve using an anteromedial thigh free flap. Based on histopathological analysis, the patient was diagnosed with basal cell adenocarcinoma (BCAC), and immunohistochemistry confirmed that the lesion was CD117, p63, SMMHC (smooth muscle myosin heavy chain), and p40 positive (Fig. 3). 

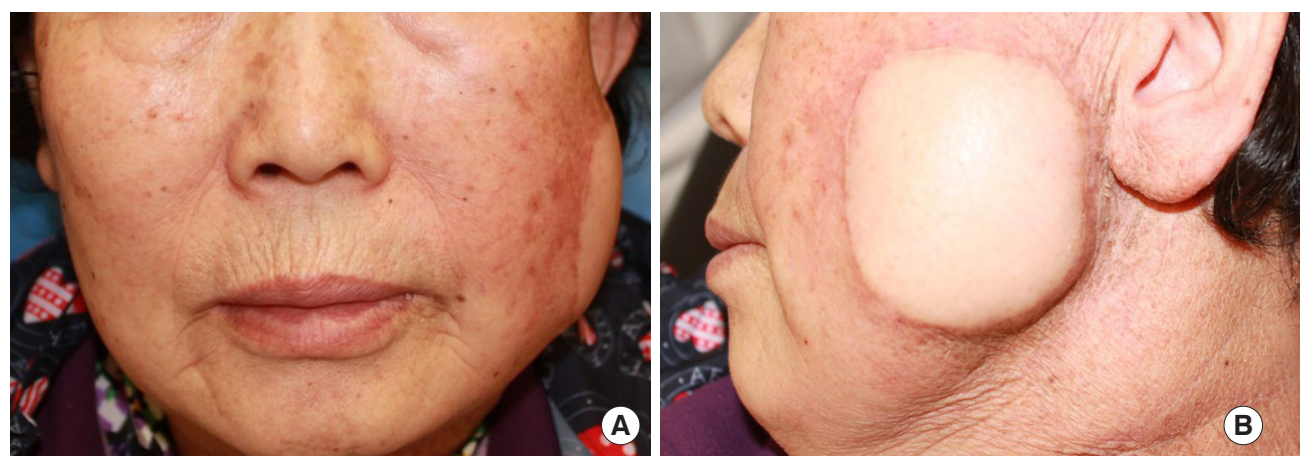

Fig. 4. Clinical photographs 12 months after surgery. (A) Anterior view and (B) lateral view.

The patient underwent radiotherapy thereafter. At the 12month follow-up, the patient had not experienced recurrence or metastasis, and the operative wound was stable (Fig. 4).

\section{DISCUSSION}

There may be occasional development of multiple tumors in the salivary glands, which account for $1.7 \%$ to $5 \%$ of parotid lesions [3]. The case we encountered can be explained by the concept of extremely rare multiple tumor development involving the metachronous onset of two distinct primary malignant tumors, EMC and BCAC, arising from the same location with a 20-year gap between the two onsets. Nonetheless, the medical records from the initial EMC were incomplete, and there may have been residual gland tissue despite total parotidectomy. Metachronous development of BCAC 20 years later most likely originated from this previous lesion. Although there is a record of total parotidectomy performed 20 years ago at another hospital record, it is not clear exactly whether it was completely resected due to lack of data. However, if the pathology of the tumor that occurred 20 years later showed a tumor with a new character, it could be assumed that there was no remnant lesion of tumor but a new tumor occurred in the same location.

The most common forms of these tumors exhibiting a histological combination include Warthin tumors and pleomorphic adenoma [4]. These multiple tumors, demonstrating two distinct characteristics from a unilateral parotid neoplasm, can be differentiated into either synchronous or metachronous tumors [5]. While synchronous tumors are characterized by the development of more than one tumor at the same time, these tumors are separated by tumor-free salivary gland tissue [6]. In these cases, the tumor may be easily confused with a hybrid tumor, which is different because this tumor has identical origin at the same topographical area but exhibits $>2$ different histological types [7]. Meanwhile, metachronous tumors arise at different time points, although there is no clearly established standard for time periods. Previous studies have suggested that for multiple tumors, metachronous (30\%) tumors are much less common than synchronous (70\%) tumors [4]. Franzen et al. [4] reviewed 796 medical records of 758 patients who underwent parotidectomy over a 40 year-period, and reported that $13 \%$ of these patients had multiple tumors. These tumors were more often unilateral than bilateral, and synchronous than metachronous. The most common forms observed were unilateral and synchronous Warthin tumors and pleomorphic adenomas. All cases of metachronous tumors were found to be contralateral.

Among tumors originating from the salivary gland(s), EMC and BCAC are rarely observed low-grade malignant tumors, which often arise from the parotid gland. In 1991, these were classified as independent diseases under the World Health Organization classification [8]. EMC has biphasic pathological characteristics involving variable proportions of two distinct cell types. More specifically, the inner layer of ductal-lining exhibits characteristics of epithelial-type cells while the outer layer exhibits characteristics of clear, myoepithelial type cells. In this tumor, local recurrence rates is ranging from $23 \%$ to $80 \%$, an incidence of metastasis is reported to be about $14 \%$ and a death rate is reported to be as high as $40 \%$ in literature [9]. BCAC has characteristics of basaloid cell proliferation similar to basal cell adenoma (BCA), but is different from BCA in that it exhibits necrosis or mitoses, as well as invasive activity such as capsular invasion. Wilson and Robinson [10] reported that BCAC recurred at a rate of $16.7 \%$ and uncommonly metastasized and rarely even causes death. Cases involving co-occurrence of EMC and BCAC are extremely rare. Franzen et al. [4] reported a case involving a 70-year-old man diagnosed with EMC, BCAC, and adenoid cystic carcinoma in a single mass on his right parotid gland, and another similar case involving EMC and BCAC simultaneously diagnosed in a single parotid gland tumor. However, the main difference in these previous cases is that they involved a hybrid tumor of a single mass at an identical anatomical location. 
Both BCAC and EMC do not have defined treatment guidelines due to their low prevalence. Nevertheless, we have established a treatment protocol after reviewing several case reviews. In our patient, the size of the tumor was relatively large $(4.4 \times$ $4.0 \times 1.9 \mathrm{~cm}$ ) and, thus, was relatively high stage (T3), and infiltrated the skin and buccalis muscle. Accordingly, surgical excision of the tumor was performed. In addition, because both tumors are reported to have high risks for locoregional recurrence, despite the low risk for metastasis, additional radiation therapy was performed. To date, after 12 months of follow-up, the patient does not exhibit any signs of recurrence.

\section{NOTES}

\section{Conflict of interest}

No potential conflict of interest relevant to this article was reported.

\section{Ethical approval}

The study was approved by the Institutional Review Board of Catholic Medical Center (IRB No. KC19ZESI0185) and performed in accordance with the principles of the Declaration of Helsinki. Written informed consent was obtained.

\section{Patient consent}

The patient provided written informed consent for the publication and the use of her images.

\section{ORCID}

Hyo Jeong Kwon https://orcid.org/0000-0002-5778-5692

Seong Ae Kim https://orcid.org/0000-0002-4457-8416

Jong Won Rhie https://orcid.org/0000-0002-9398-8059

Suk-Ho Moon https://orcid.org/0000-0003-1866-2910

\section{REFERENCES}

1. Whitt JC, Schafer DR, Callihan MD. Multiple malignant salivary gland neoplasms: mucoepidermoid carcinoma of palate and adenoid cystic carcinoma of floor of mouth. Head Neck Pathol 2008;2:41-8.

2. Curry JL, Petruzzelli GJ, McClatchey KD, Lingen MW. Synchronous benign and malignant salivary gland tumors in ipsilateral glands: a report of two cases and a review of literature. Head Neck 2002;24:301-6.

3. Ochal-Choinska A, Bruzgielewicz A, Osuch-Wojcikiewicz E. Synchronous multiple unilateral parotid gland tumors of benign and malignant histological types: case report and literature review. Braz J Otorhinolaryngol 2019;85:388-92.

4. Franzen AM, Coordes A, Franzen CK, Guenzel T. Are multiple tumors of the parotid gland uncommon or underestimated? Anticancer Res 2017;37:5263-7.

5. Ethunandan M, Pratt CA, Morrison A, Anand R, Macpherson DW, Wilson AW. Multiple synchronous and metachronous neoplasms of the parotid gland: the Chichester experience. Br J Oral Maxillofac Surg 2006;44:397-401.

6. Bae YK, Kim DS, Suh JS, Ro JY. Combined epithelial-myoepithelial carcinoma and basal cell adenocarcinoma of the parotid gland: a case report. Korean J Pathol 1999;33:453-6.

7. Sabri A, Bawab I, Khalifeh I, Alam E. Hybrid tumor of the parotid gland: a case report and review of the literature. Case Rep Otolaryngol 2015;2015:192453.

8. Zhan KY, Lentsch EJ. Basal cell adenocarcinoma of the major salivary glands: a population-level study of 509 cases. Laryngoscope 2016;126:1086-90.

9. Gore MR. Epithelial-myoepithelial carcinoma: a populationbased survival analysis. BMC Ear Nose Throat Disord 2018;18: 15.

10. Wilson TC, Robinson RA. Basal cell adenocarcinoma and Basal cell adenoma of the salivary glands: a clinicopathological review of seventy tumors with comparison of morphologic features and growth control indices. Head Neck Pathol 2015;9: 205-13. 